\title{
Emerging role of microRNAs in allergic diseases
}

\author{
Faoud Ishmael $\left.\right|^{1,2}$ \\ 'Departmen of Allergy and Sleep Medicine, Mount Nittany Medical Group, State College, PA 16803, USA. \\ 2Department of Medicine, Section of Allergy and Immunology, Pennsylvania State University Hershey Medical Center, Hershey, PA 17033, \\ USA.
}

Correspondence to: Dr. Faoud Ishmael, Department of Allergy and Sleep Medicine, Mount Nittany Medical Group, 1850 E. Park Ave, Suite 201, State College, PA 16803, USA. E-mail: faoud.ishmael@mountnittany.org

How to cite this article: Ishmael E. Emerging role of MicroRNAs in allergic diseases. J Trans/ Genet Genom 2019;3:6. https://doi.org/10.20517/jtgg.2018.32

Received: 6 Dec 2018 Accepted: 25 Feb 2019 Published: 14 Mar 2019

Science Editor: Faoud Terrence Ishmael Copy Editor: Cai-Hong Wang Production Editor: Huan-Liang Wu

The prevalence of allergic diseases has risen at alarming rates, and a recent study identified allergic sensitization in $40 \%$ of school children worldwide ${ }^{[1]}$. Allergic diseases affect a wide variety of organs, including eyes (allergic conjunctivitis), nose (allergic rhinitis), airway (asthma), gastrointestinal tract (food allergies and eosinophilic esophagitis), and skin (atopic dermatitis). Common gaps among these diseases are the lack of understanding of the molecular basis of pathogenesis (particularly how and why inflammation is de-regulated), and the crucial need to identify biomarkers to better diagnose and characterize these diseases. Along these lines, microRNAs (miRNAs) have emerged as central regulators of many processes (including inflammation) and potentially useful biomarkers (in large part because they are found in all biofluids). As a result, it is not surprising that these two fields have intersected, and a better understanding of how miRNAs regulate allergic inflammation could lead to novel therapies and diagnostic tools.

We are just beginning to understand the roles of miRNAs in allergic diseases. This special issue highlights the emerging roles of miRNAs in across a spectrum of allergic disease that affects different organ systems, and demonstrates their potential application to understanding, treating, and diagnosing human disease. The article by Weidner et al ${ }^{[2]}$ reviews the progress in our understanding of miRNAs in asthma, and the evolution of research from mouse to humans. This review captures the translational research potential of miRNAs in asthma, and underscores the need for both mouse and human studies in mechanistic miRNA studies. In particular, ablation of pathogenic miRNAs (such as miR-155) in mice has demonstrated their crucial role in pathogenesis of allergic inflammation, and their conserved role in human disease is now becoming evident. In work from our lab, Zhang et al. ${ }^{[3]}$ present a primary research article that builds on the mechanistic studies to show how miRNAs can be used to better characterize asthma. We now understand that asthma is a syndrome that is comprised of many distinct phenotypes, which have different molecular

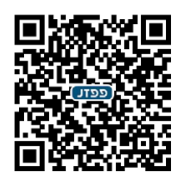


causes and respond differently to medications. There is a lack of biomarkers to categorize patients into these phenotypes, which hampers our ability to effectively diagnose and treat asthma. Our work demonstrated that blood miRNAs are capable of objectively categorizing patients in different phenotypes, which may tell us about the molecular mechanisms of these forms of asthma and allow us to better tailor treatment for each patient (personalized therapy) ${ }^{[3]}$. It is interesting to note that many of the miRNAs discussed by Weidner et al. ${ }^{[2]}$ were candidates we identified in the biomarker study, indicating that these are functional biomarkers that may also serve as therapeutic targets.

The article by Bhardwaj further extends the miRNA research to atopic dermatitis, an allergic skin disease ${ }^{[4]}$. Many of the asthma candidate miRNAs emerged as players in the pathogenesis of atopic dermatitis, suggesting that miRNA pathways may be de-regulated in the skin in an analogous manner to the airways in asthma. Along these lines, Lambert et al. ${ }^{[5]}$ reviewed the current literature on miRNAs in eosinophilic esophagitis, a relatively new disease whose pathogenesis is poorly characterized. A panel of miRNAs was found to be de-regulated in inflamed esophageal tissue, and mechanistic studies suggest that they regulate allergic cytokine signaling. Together, these studies demonstrate the potential importance of miRNAs as pathogenic mediators and biomarkers of allergic disease. Translational approaches using mouse models and humans have produced findings that have direct clinical relevance. Many miRNAs were implicated in common across organ-specific allergic diseases, suggesting that targeting them for therapeutics could present a strategy to treat a broad spectrum of allergic diseases.

\section{DECLARATIONS}

\section{Authors' contributions}

The author contributed solely to the article.

\section{Availability of data and materials}

Not applicable.

\section{Financial support and sponsorship}

None.

\section{Conflicts of interest}

The author declared that there are no conflicts of interest.

\section{Ethical approval and consent to participate}

Not applicable.

\section{Consent for publication}

Not applicable.

\section{Copyright}

(c) The Author(s) 2019.

\section{REFERENCES}

1. Pawankar RS, Sanchez-Borges M, Bonini S, Kaliner MA. The burden of allergic diseases. In: Pawankar R, Canonica GW, Holgate ST, Lockey RF, editors. White book on allergy 2011-2012 executive summary. Milwaukee: World Allergy Organization; 2011. pp. 27-74.

2. Weidner J, Malmhäll C, Rådinger M. microRNAs in asthma pathogenesis - from mouse to man. J Transl Genet Genom 2019;3:2.

3. Zhang S, Laryea Z, Panganiban R, Lambert K, Hsu D, et al. Plasma microRNA profiles identify distinct clinical phenotypes in human asthmatics. J Transl Genet Genom 2018;2:18.

4. Bhardwaj N. MicroRNAs in atopic dermatitis: a review. J Transl Genet Genom 2017;1:15-22.

5. Lambert KA, Jhaveri P, Jhaveri P. Biomarkers and therapeutic targets: microRNA roles in the pathophysiology, diagnosis and management of eosinophilic esophagitis. J Transl Genet Genom 2018;2:11. 